\title{
Deletion of GRK1 Causes Retina Degeneration through a Transducin-Independent Mechanism
}

\author{
Jie Fan, ${ }^{1 \star}$ Keisuke Sakurai, ${ }^{3 \star}$ Ching-Kang Chen, ${ }^{4}$ Baerbel Rohrer,,${ }^{1,2}$ Bill X. Wu, ${ }^{1}$ King-Wai Yau, ${ }^{5}$ Vladimir Kefalov, ${ }^{3}$ \\ and Rosalie K. Crouch ${ }^{1}$ \\ Departments of ${ }^{1}$ Ophthalmology and ${ }^{2}$ Neuroscience, Division of Research, Medical University of South Carolina, Charleston, South Carolina 29412, \\ ${ }^{3}$ Department of Ophthalmology and Visual Sciences, Washington University, St. Louis, Saint Louis, Missouri 63110, ${ }^{4}$ Department of Biochemistry and \\ Molecular Biology, Virginia Commonwealth University, Richmond, Virginia 23298, and ${ }^{5}$ Department of Neuroscience, Johns Hopkins University School of \\ Medicine, Baltimore, Maryland 21205
}

\begin{abstract}
Rpe65 $5^{-I-}$ mice are unable to produce 11-cis-retinal, the chromophore of visual pigments. Consequently, the pigment is present as the apoprotein opsin with a minute level of pigment containing 9-cis-retinal as chromophore. Notably, a 10-20\% fraction of this opsin is mono-phosphorylated independently of light conditions. To determine the role of rhodopsin kinase (GRK1) in phosphorylating this opsin and to test whether eliminating this phosphorylation would accelerate photoreceptor degeneration, we generated the $R p e 65^{-I-}$ Grk $1^{-I-}$ mouse. The retinae of Rpe $65^{-I-} \mathrm{Grk1} 1^{-l-}$ mice had negligible opsin phosphorylation, extensive degeneration with decreased opsin levels, and diminished light-evoked rod responses relative to Rpe65 $5^{-I-}$ mice. These data show that opsin phosphorylation in the Rpe $65^{-1-}$ mouse is due to the action of GRK1 and is neuroprotective. However, despite the higher activity of unphosphorylated opsin, the severe loss of opsin in the rapidly degenerating Rpe $65^{-1-} \mathrm{Grk1} 1^{-1-}$ mice resulted in lower overall opsin activity and in higher rod sensitivity compared with Rpe $65^{-1-}$ mice. In Rpe $65^{-1-}$ Grk $1^{-1-}$ Gnat $1^{-1-}$ mice where transduction activation was blocked, degeneration was only partially prevented. Therefore, increased opsin activity in the absence of phosphorylation was not the only mechanism for the accelerated retinal degeneration. Finally, the deletion of GRK1 triggered retinal degeneration in Grk ${ }^{-/-}$mice after 1 month, even in the absence of apo-opsin. This degeneration was independent of light conditions and occurred even in the absence of transducin in Grk1 ${ }^{-l-}$ Gnat $1^{-l-}$ mice. Taken together, our results demonstrate a light-independent mechanism for retinal degeneration in the absence of GRK1, suggesting a second, not previously recognized role for that kinase.
\end{abstract}

\section{Introduction}

Vision is initiated when light is absorbed by visual pigments, triggering the isomerization of 11-cis-retinal to the all-trans isomer, resulting in activation of the visual cascade. The lifetime of the activated pigment is limited by a two-step process: active rhodopsin is first phosphorylated by rhodopsin kinase (GRK1) and then becomes completely inactivated when visual arrestin

\footnotetext{
Received Dec. 17, 2009; accepted Jan. 9, 2010.

This work was supported by National Institutes of Health (NIH) Grants EY04939 (R.K.C.), EY13520 (B.R.), EY13811 (C.K.C.), EY06837 (K.-W.Y.), EY19312 (V.J.K.), EY14793 [Medical University of South Carolina (MUSC) vision core], and EY02687 (Department of Ophthalmology and Visual Sciences, Washington University), Career Development Award from Research to Prevent Blindness (RPB) (New York, NY) and Karl Kirchgessner Foundation (V.J.K.), and an unrestricted grant to the Department of Ophthalmology at MUSC from RPB. R.K.C. is an RPB Senior Scientific Investigator, and B.R. is an RPB Olga Keith Weiss Scholar. The Medical University of South Carolina animal studies were conducted in a facility constructed with support from the Research Facilities Program, Grant C06 RR015455 from the NIH National Center for Research Resources. We thank Michael Redmond, National Eye Institute at NIH, for the gift of the Rpe65 ${ }^{-1-}$ mice, and Zsolt Ablonczy, MUSC, for assistance with the phosphorylation measurements. We also thank Gordon Fain and Michael Woodruff, University of California, Los Angeles, for their heroic attempts to conduc single-cell physiology on these animals, and Clint Makino, Masahiro Kono, Yiannis Koutalos, Vladlen Slepak, and Anita Zimmerman for helpful discussions.

*J.F. and K.S. contributed equally to this work.

Correspondence should be addressed to Jie Fan, Department of Ophthalmology, Medical University of South Carolina, 167 Ashley Avenue, Charleston, SC 29425. E-mail: fan@musc.edu.

D01:10.1523/JNEUROSCI.6254-09.2010

Copyright $\odot 2010$ the authors $\quad 0270-6474 / 10 / 302496-08 \$ 15.00 / 0$
}

binds (Arshavsky, 2002). Phosphorylation is a vital first step in the timely termination of phototransduction.

In vitro, rhodopsin phosphorylation has been shown to be catalyzed by both GRK1 (Ohguro et al., 1996; Chen et al., 1999) and protein kinase C (PKC) (Newton and Williams, 1993; Williams et al., 1997). In vivo, the role of GRK1 in terminating phototransduction has been clearly demonstrated by studies on the $G r k 1^{-1-}$ mouse (Chen et al., 1999) and by deleting the rhodopsin phosphorylation sites (Chen et al., 1995; Mendez et al., 2000). Phosphorylation of rhodopsin is the only reported role for GRK1 in the retina. PKC is also expressed in rod outer segments (Kelleher and Johnson, 1986; Williams et al., 1997) and has been proposed to have a role in rhodopsin phosphorylation. However, no effect on rod phototransduction was observed on the use of PKC activators or inhibitors (Xiong et al., 1997) and Adams et al. (2003) concurred that GRK1 is the main enzyme for rhodopsin phosphorylation. The phosphorylation target of $\mathrm{PKC}$ in photoreceptors is still unclear.

The Rpe65 ${ }^{-1-}$ mouse does not form 11-cis-retinal in the retinal pigment epithelium (Redmond et al., 1998). Consequently, the majority of visual pigment in $R p e 65^{-1-}$ rods is in the form of the apoprotein, opsin, together with minute amounts $(<0.1 \%)$ of isorhodopsin (Fan et al., 2003). The rods in Rpe65 ${ }^{-1-}$ mice degenerate slowly (Fan et al., 2005), and this degeneration can be blocked by ablating the $\alpha$-transducin gene, Gnat1 (Woodruff et 


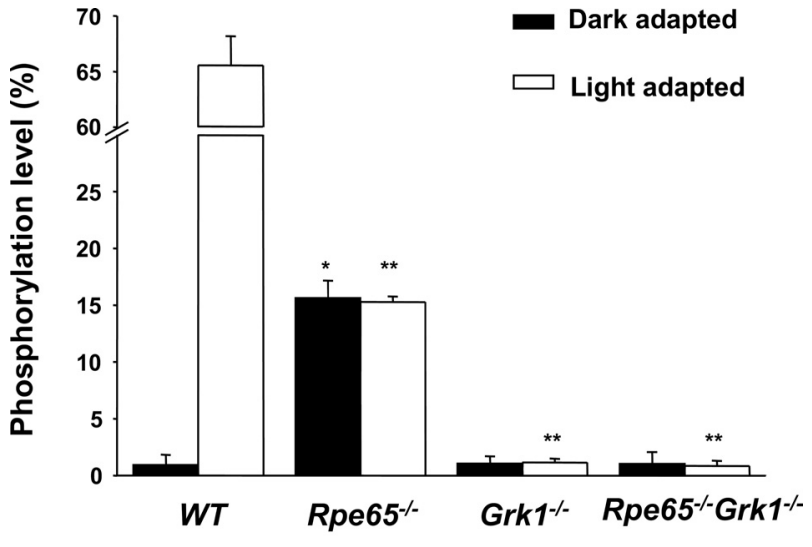

\section{2-month-old mice}

Figure 1. Effect of deletion of Rpe65 and Grk1 on opsin phosphorylation. Retinae of cycliclight-reared, 2-month-old WT, Rpe65 $5^{-1-}$, Grk1 ${ }^{-1-}$, and Rpe65 $5^{-1-} \mathrm{Grk1} 1^{-1-}$ mice were homogenized in $8 \mathrm{~m}$ urea and digested with endoproteinase Asp-N in $10 \mathrm{~mm}$ Tris buffer at pH 7.6 to cleave the opsin C terminus, which was analyzed online with an LCQ mass spectrometer. In the $G r k 1^{-1-}$ and Rpe65 $5^{-1-}$ Grk $1^{-1-}$ mice, no significant opsin phosphorylation was observed. White bars, Animals exposed to room light for $6 \mathrm{~h}$; black bars, animals dark-adapted for $12 \mathrm{~h}$. Data are shown as the percentage of rhodopsin C terminus containing phosphorylation, independent of the multiplicity of phosphorylation, and presented as mean $\pm \mathrm{SEM} ; n=3$.

A

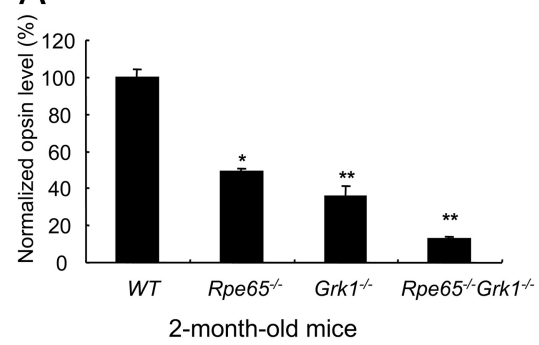

C

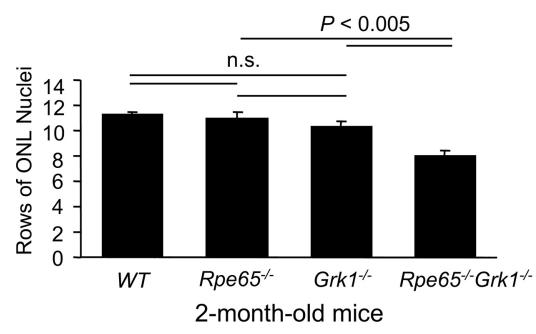

B

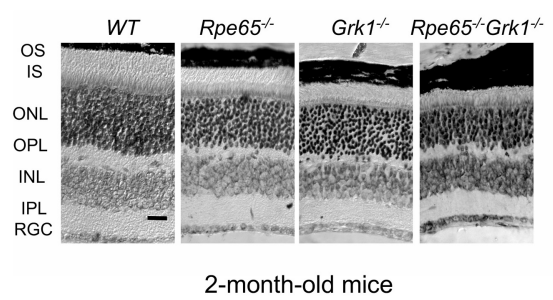

D $\quad$ DT $\square$ Rpe65 $\square$ Grk $1 \% \quad$ 圈 Rpe65 $\%$ Grk $1 \%$

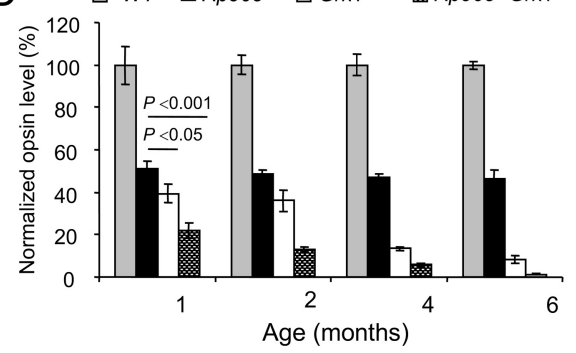

Figure 2. Effect of deletion of Rpe65 and Grk1on retinal opsin levels and morphology. A, Opsin levels were calculated from rhodopsin that formed upon the addition of 11-cis-retinal. Data were generated from 2-month-old cyclic-light-reared mice. The relative opsin levels in each strain were normalized to the average of wild-type opsin concentration and shown as a fraction of the wild type. B, Retinal morphology of 2-month-old WT, Rpe65 ${ }^{-1-}, G r k 1^{-1-}$, and Rpe65 $5^{-1-} \mathrm{Grk} 1^{-1-}$ mice. Light micrographs of paraffin-embedded retinae sections from the superior central region of the retina. While morphology of the inner retina was unaffected by the different genotypes at that resolution, the outer retina showed shortening of the inner and outer segments progressing in severity from the Rpe65 $5^{-1-}<\mathrm{Grk1}^{-1-} \ll R p e 65^{-1-}$ Grk1 ${ }^{-1-}$ (scale bar, $30 \mu \mathrm{m}$ ). INL, Inner nuclear layer; IPL, inner plexiform layer; IS, inner segments; OPL, outer plexiform layer; OS, outer segments; RGC, retinal ganglion cells; $n . s$. , not significant. C, Bar graph representing rows of photoreceptor cell nuclei counted in the superior central region of the retinae of 2-month-old mice from the 4 strains; mean $\pm \mathrm{SEM} ; n=5$. The cell loss was significant for the Rpe65 ${ }^{-I-}$ Grk ${ }^{-I-}$ mice. D, Opsin levels with age, assayed as for $\boldsymbol{A}$. The relative opsin levels in each strain were normalized to the average of wild-type opsin concentration at each age, and shown as the fraction of the wild type. Gray bar, WT mice; black bar, Rpe65 ${ }^{-1-}$ mice; white bar, Grk $1^{-1-}$ mice; hatched bar, Rpe65 ${ }^{-1-}$ Grk $1^{-1-}$ mice.

al., 2003), indicating that degeneration occurs due to the constitutive activation of the rod signal transduction cascade by the opsin. Notably, the rod opsin in the Rpe65 $1-1-$ mouse as well as in the $\mathrm{Lrat}^{-1-}$ mouse, which also lacks 11-cis-retinal

\section{Materials and Methods}

Animals. Rpe65 ${ }^{-1-}$ (B6;129-Rpe65 $\left.{ }^{\mathrm{tm} 1 \mathrm{Tmr}}\right)$ mice were the generous gift of Dr. Michael Redmond (National Eye Institute, National Institutes of Health) and genotyped as described previously (Redmond et al., 1998; Redmond and Hamel, 2000). Grk $1^{-1-}$ mice (B6;129-Grk $\left.1^{\text {tm1Mis }}\right)$ were generated as described previously (Chen et al., 1999). Rpe65 ${ }^{-1-}$ Grk1 $1^{-1-}$ mice (B6;129Rpe $65^{\text {tmlTmr }}$ Grk1 ${ }^{\text {tmlMis/Kwy }}$ ) were generated by cross-breeding the Rpe65 $5^{-I-}$ mice with $G r k 1^{-1-}$ mice. Western blot analysis confirmed that GRK1 and RPE65 were not detectable in homogenates of eyecups from Rpe65 ${ }^{-/-} \mathrm{Grk1} 1^{-/-}$ mice. Gnat $1^{-/-}$mice (B6;129-Gnat $\left.1^{\mathrm{tmlJl}}\right)$ were the generous gift of Dr. Janis Lem (New England Medical Center and Tufts University School of Medicine, Boston, MA). Rpe65 $5^{-1-}$ $\mathrm{Grk1}^{-1-}$ Gnat $^{-1-}$ mice were generated by cross-breeding the Rpe $65^{-1-}$ Grk1 $1^{-/-}$mice and Gnat $1^{-1-}$ mice and genotyped as described previously (Calvert et al., 2000). Western blot analysis confirmed the genotype results. Age-matched C57BL/6 (WT) mice were purchased from Harlan. Animals were reared under cyclic light ( $12 \mathrm{~h}$ light/12 h dark) with the ambient light intensity at the eye level being $85 \pm 18$ lux until experimentation. For some experiments, mice were raised under constant darkness from birth with animal husbandry performed under very dim red light (0.3 lux). All experiments were performed in accordance with the policy on the Use of Animals in Neuroscience Research and were approved by the Medical University of South Carolina Animal Care and Use Committee and the Washington University Animal Studies Committee.

Opsin quantification. Retinae from darkadapted mice ( $12 \mathrm{~h}$ ) were collected under infrared light. A pair of retinae was homogenized with a glass syringe in 500 $\mu \mathrm{l}$ of $10 \mathrm{~mm}$ Tris- $\mathrm{HCl}$ containing $1 \mathrm{~mm}$ EDTA (pH 7.5), AEBSF [ $1 \mathrm{~mm}$ 4-(2-aminoethyl)-benzene sulfonyl fluoride hydro-chloride; Roche Molecular Biochemicals], protease inhibitor mixture tablet $(1 \mathrm{tablet} / 10 \mathrm{ml}$; 
Complete Mini; Roche Molecular Biochemicals), and $10 \mu \mathrm{g}$ of DNase I (Sigma). Samples were centrifuged $(27,000 \times g, 15 \mathrm{~min})$, and the supernatant was discarded. The pellets were suspended in $100 \mu \mathrm{l}$ of $0.1 \mathrm{M}$ sodium phosphate buffer ( $\mathrm{pH}$ 7.4) and incubated with 11-cisretinal (final concentration, $80 \mu \mathrm{M}$ ) at $4^{\circ} \mathrm{C}$ on a rotator for $12 \mathrm{~h}$. Samples were centrifuged, and the resultant pellets were resuspended in $100 \mu \mathrm{l}$ of $1 \% n$-dodecyl- $\beta$-D-maltoside (ULTROL grade; Calbiochem) in $0.1 \mathrm{~m}$ sodium phosphate buffer ( $\mathrm{pH}$ 7.4) for solubilization $\left(2 \mathrm{~h}, 4^{\circ} \mathrm{C}\right.$ on a rotator). Unsolubilized material was removed by centrifugation $(100,000 \times g$ for $15 \mathrm{~min}$ ), and the supernatant was analyzed by spectrophotometry (Cary 300, Varian). Samples were exposed to white light (Fiber Optic Illuminator, Model 190, $50 \mathrm{~W}, 60 \mathrm{~Hz}$; Dolan-Jenner Industries) for $10 \mathrm{~min}$ in the presence of hydroxylamine hydrochloride $(\mathrm{pH}$ 7.0, $20 \mathrm{~mm}$ final concentration). Pigment levels were determined by subtracting the postbleach from the prebleach spectra. Rhodopsin concentrations were calculated using the extinction coefficient of $40,000 \mathrm{M}^{-1} \mathrm{~cm}^{-1}$ (Wald and Brown, 1958; Dartnall, 1968).

Transcorneal ERG recordings. Overnight dark-adapted mice were anesthetized using xylazine $(20 \mathrm{mg} / \mathrm{kg}$, i.p. $)$ and ketamine $(80 \mathrm{mg} / \mathrm{kg}$, i.p.). Pupils were dilated with phenylephrine hydrochloride $(2.5 \%)$ and atropine sulfate $(1 \%)$. Contact lens electrodes (Bayer et al., 2001) were placed on both eyes accompanied by $2.5 \%$ Gonak hypromellose ophthalmic demulcent solution. Full-field ERGs were recorded as described previously (Gresh et al., 2003), using the universal testing and electrophysiologic system 2000 (UTAS E-2000; LKC Technologies). Single flashes of $10 \mu$ s duration and various intensities $\left(2.48 \times 10^{-2}, 2.48 \times 10^{-1}, 1.56\right.$ and $2.48 \mathrm{~cd}^{\star} \mathrm{s} / \mathrm{m}^{2}$ ) were used for stimulation under scotopic conditions.

Transretinal ERG recordings. WT, Rpe65 ${ }^{-1-}$, and Rpe $65^{-1-} \mathrm{Grk1}^{-1-}$ mice were reared in 12/12 h light/dark cycle and were dark-adapted overnight before experiments. To slow down retinal degeneration, $G r k 1^{-1-}$ mice and a subpopulation of Rpe65 $5^{-1-} \mathrm{Grk1} 1^{-1-}$ mice were reared in constant darkness from birth. Recordings were made in mice approximately 1 month old. Following killing, the eyes were removed under dim red light. All subsequent manipulations were done under infrared light. The eye was hemisected, and the retina was isolated and stored in Locke solution at $4^{\circ} \mathrm{C}$. One-quarter of the isolated retina was mounted on filter paper with the photoreceptor side up and placed on the recording chamber with an electrode connected to the bottom. A second electrode was placed above the retina. The perfusing Locke solution (112 mM NaCl, $3.6 \mathrm{~mm} \mathrm{KCl,} 2.4 \mathrm{mM} \mathrm{MgCl}_{2}, 1.2 \mathrm{mM} \mathrm{CaCl}_{2}$, $10 \mathrm{~mm}$ HEPES, $20 \mathrm{~mm} \mathrm{NaHCO}_{3}, 3 \mathrm{~mm} \mathrm{Na}_{2}$-succinate, $0.5 \mathrm{~mm} \mathrm{Na}$ glutamate, $10 \mathrm{~mm}$ glucose) was equilibrated with $95 \% \mathrm{O}_{2} / 5 \% \mathrm{CO}_{2}$, heated to $34-37^{\circ} \mathrm{C}$, and contained, in addition, $2 \mathrm{~mm} \mathrm{~L}$-glutamic acid to block higher order components of the photoresponse (Sillman et al., 1969). The electrode solution ( $140 \mathrm{~mm} \mathrm{NaCl}, 3.6 \mathrm{~mm} \mathrm{KCl}, 2.4 \mathrm{~mm} \mathrm{MgCl}$, $1.2 \mathrm{~mm} \mathrm{CaCl}_{2}, 3$ mм HEPES, $10 \mathrm{~mm}$ glucose, $\mathrm{pH}$ 7.4) under the retina contained, in addition, $10 \mathrm{~mm} \mathrm{BaCl}_{2}$ to suppress the glial component of the photoresponse (Bolnick et al., 1979; Nymark et al., 2005). Responses were amplified by a differential amplifier (DP-311; Warner Instruments). For application of 11-cis-retinal, an isolated retina was incubated

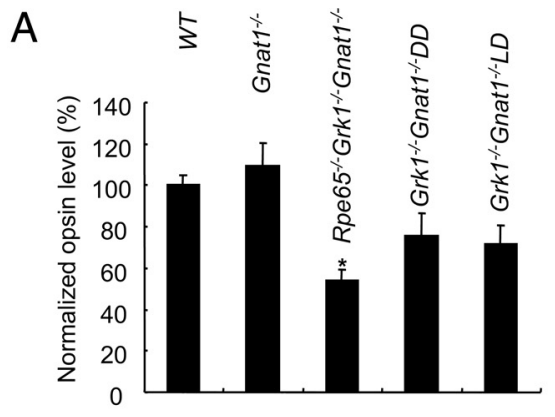

2-month-old animals

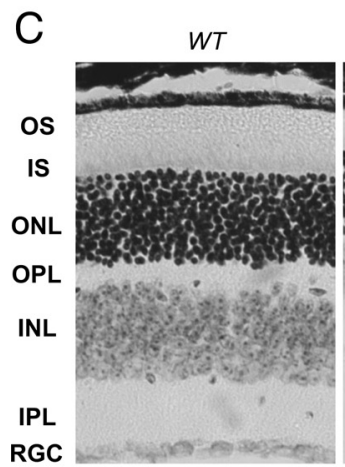

Figure 3. Effect of deletion of Gnat 1 on photoreceptor degeneration in the absence of Grk1. A, Opsin levels were calculated as for Figure 2A. Data are for 2-month-old mice. The relative opsin levels were normalized to the average of wild-type opsin concentration and shown as the fraction of wild type. $B, 0$ psin levels from Rpe65 ${ }^{-1-} \mathrm{Grk} 1^{-/-} \mathrm{Gnat1} 1^{-I-}$ mice with age, assayed Light micrographs of paraffin-embedded retinae of 6-month-old cyclic-light-reared animals were taken from the superior centra egion of the eye to compare the thicknesses of the different layers. Note the extreme degeneration in the Rpe65 ${ }^{-I-} \mathrm{Grk} 1^{-I-}$ retina. Scale bar, $20 \mu \mathrm{m}$. INL, Inner nuclear layer; IPL, inner plexiform layer; IS, inner segments; OPL, outer plexiform layer; OS, outer segments; RGC, retinal ganglion cells.

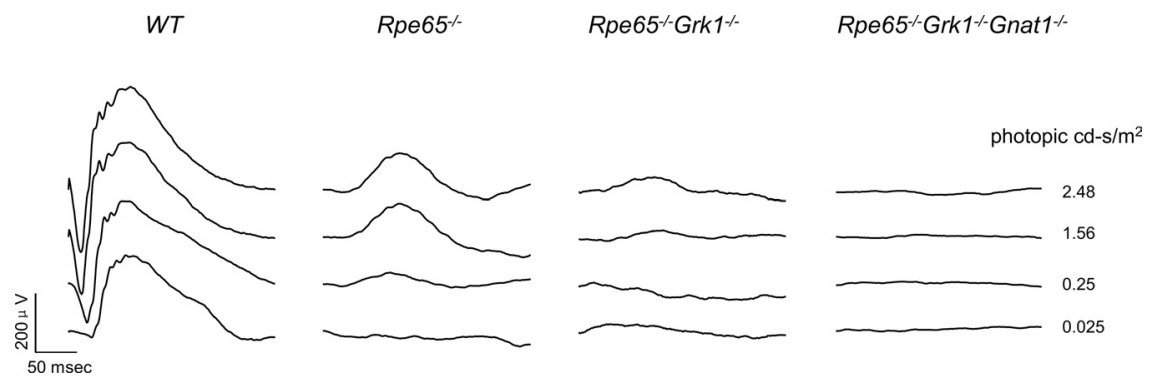
Figure 4. Scotopictranscorneal ERG responses. Families of single-flash ERGs were recorded from 2-month-old WT, Rpe65 $5^{-/-}$,
and Rpe65 ${ }^{-I-} \mathrm{Grk} 1^{-1-}$ mice in response to increasing light intensities $2.48 \times 10^{-2}, 2.48 \times 10^{-1}, 1.56$ and $2.48 \mathrm{~cd}^{*} \mathrm{~s} / \mathrm{m}^{2}$.

in electrode solution containing $100 \mu \mathrm{M}$ 11-cis-retinal (dissolved in $0.1 \%$ ethanol) and $1 \%$ bovine serum albumin at room temperature for $1.5 \mathrm{~h}$ before recording. Due to the low light sensitivity of Rpe65 $5^{-1-} \mathrm{Grk1} 1^{-1-}$ rods and to be able to compare sensitivity between genotypes with a wide range of sensitivity, white light rather than $510 \mathrm{~nm}$ monochromatic light was used for photostimulation. White test flashes were delivered from a calibrated light source via computer-controlled shutters. For attenuated light, the flash intensity was set by a combination of neutral-density filters. To expand the stimulation range for unattenuated light, the flash duration was increased sequentially from the standard $10 \mathrm{~ms}$ to 20,40 , and $80 \mathrm{~ms}$.

Intensity-response data were fitted by Equation 1, as follows:

$$
\frac{R}{R_{\max }}=\frac{I^{n}}{I^{n}+I_{o}^{n}}
$$



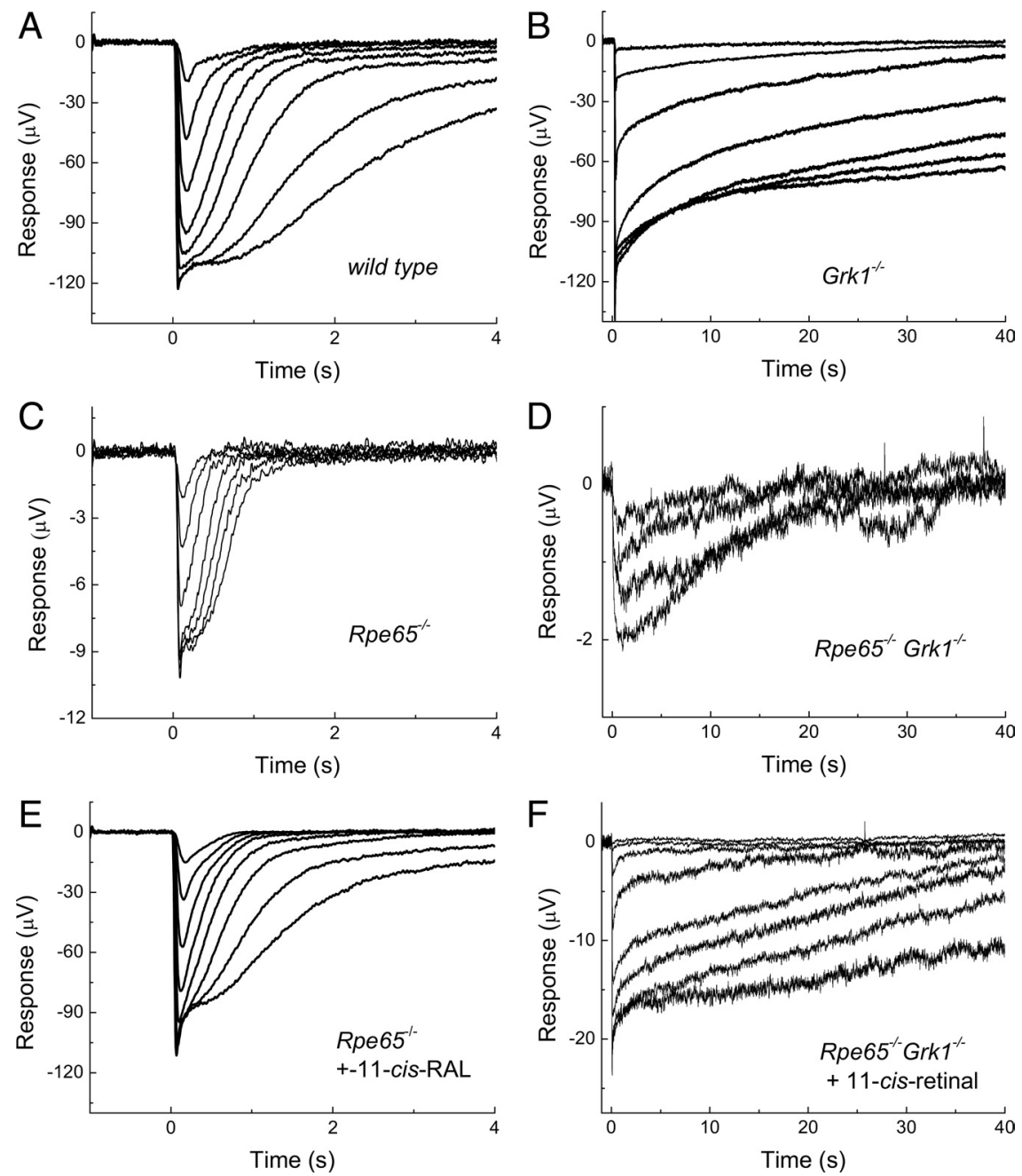

Figure 5. $\boldsymbol{A}-\boldsymbol{F}$, Response families of transretinal ERG recordings from WT $(\boldsymbol{A}), \mathrm{Grk1} 1^{-1-}(\boldsymbol{B})$, Rpe65 $^{-1-}(\boldsymbol{C}, \boldsymbol{E})$, and Rpe65 $5^{-1}$ $-G r k 1^{-1-}(\boldsymbol{D}, \boldsymbol{F})$ mice. Responses in $\boldsymbol{E}$ and $\boldsymbol{F}$ were recorded after application of 11-cis-retinal. Flashes of white light were delivered at time 0 in 0.5 log incremental steps except for the last three traces in $C$, which were delivered in twofold increments by doubling the flash duration. The dimmest flashes were delivered at $-1.5 \log (C, D)$, and $-4.5 \log (A, B, E$, and $F)$ unit attenuation.

where $R$ is the transient-peak amplitude of response, $R_{\max }$ is maximal response amplitude, $I$ is flash intensity, and $I_{o}$ is flash intensity estimated to produce half-maximal response. Comparison of sensitivity measured with white light and with calibrated $500 \mathrm{~nm}$ light in wild-type retina revealed that the intensity of unattenuated white light at 10-ms duration was equivalent to $2.8 \times 10^{5}$ photons $\mu \mathrm{m}^{-2}$ at $500 \mathrm{~nm}$. To describe the kinetics of the dim-flash response, the time-to-peak $\left(T_{\text {peak }}\right)$ was determined as the time from the mid-point of the test flash to the peak of the response, and the recovery time constant $\left(\tau_{\text {rec }}\right)$ was determined by fitting the final response decline with a single-exponential-decline function.

Histology. The eyes were enucleated and immersion-fixed in a solution of $60 \%$ methanol, $30 \%$ chloroform, and $10 \%$ acetic acid overnight at $4^{\circ} \mathrm{C}$, and dehydrated over several hours before being embedded in paraffin in transverse orientation. The eyes were sectioned at $7 \mu \mathrm{m}$ thickness and mounted on poly-L-lysine-coated slides. The sections were stained with $1 \%$ toluidine blue in deionized water, dehydrated, and coverslipped with mounting medium (Permount; Fisher Scientific). Central areas of the retina (within 100-300 $\mu \mathrm{m}$ of the optic nerve) were photographed for documentation. Images were acquired on a Zeiss microscope (Axioplan 2). Cell nuclei were counted by two individuals without knowledge of the identity of the tissues.

Phosphorylation measurements. Retinae (two per sample) were homogenized in $8 \mathrm{M}$ urea and digested with Asp-N (25 ng; Sigma) in $100 \mu \mathrm{l}$ of Tris buffer ( $10 \mathrm{~mm}, \mathrm{pH}$ 7.6). Supernatants were collected by centrifugation $(120,000 \times g)$ and analyzed online with an LCQ mass spectrom- eter (Thermo-Finnigan Instrument Systems) (Hurley et al., 1998; Ablonczy et al., 2000). The data were acquired by repetitive scanning and corrected with a factor for the decreased detection efficiency of the C-terminal phosphopeptides, as measured from synthesized standards [the peak area of mono-phosphopeptides was multiplied by 1.35 (Hurley et al., 1998)].

Statistics. For all experiments, data were expressed as mean \pm SEM. Data were analyzed using a two-tailed Student $t$ test, accepting a significance level of $p<0.05$.

\section{Results}

\section{Opsin phosphorylation in Rpe65 $5^{-/-}$} mice depends on GRK1

Rod opsin in mouse models lacking 11cis-retinal, such as the Rpe65 $5^{-1-}$ and $\mathrm{Lrat}^{-1-}$ mice, is known to be mono-phosphorylated (Ablonczy et al., 2002; Fan et al., 2008). We measured the rod opsin phosphorylation levels in WT, Rpe65 ${ }^{-1-}$, Grk1 $1^{-1-}$ and Rpe $65^{-1-}$ Grk1 $1^{-1-}$ mice to determine whether GRK1 is the kinase responsible for the phosphorylation of opsin in Rpe65 $5^{-1-}$ mice. Phosphorylation was quantified by cleavage of the $\mathrm{C}$ terminus of the opsin protein followed by massspectrometric analysis (Ablonczy et al., 2002) (Fig. 1). The light-adapted WT mice showed $65.5 \pm 2.7 \%(n=3) \operatorname{rod}$ opsin phosphorylation, of which $28.6 \pm$ $1.4 \%(n=3)$ was mono-phosphorylation. There was essentially no phosphorylation in dark-adapted WT mice. The Rpe65 ${ }^{-1-}$ mice showed $15.6 \pm 1.5 \%(n=3)$ phosphorylated opsin, all mono-phosphorylated, independent of light exposure. This phosphorylation was not specific to a single site, but distributed over the major sites observed in light-activated mouse rhodopsin (serines 334, 338, and 343 with some evidence of threonine 342 ; data not shown). The $\mathrm{Grk1}^{-1-}$ mice showed no significant phosphorylation regardless of light exposure, as reported previously (Chen et al., 1999). Most important, the deletion of GRK1 in RPE65-deficient mice (Rpe65 $5^{-1-} \mathrm{Grk1}^{-/-}$) also ablated opsin phosphorylation in darkness or light. Therefore, GRK1 underlies the phosphorylation of rod opsin in Rpe65 $5^{-1-}$ mice.

\section{$R p e 65^{-1-}$ mice show rapid retinal degeneration in absence of GRK1}

To determine how the lack of opsin phosphorylation affects the survival of photoreceptors, we quantified opsin levels in 2-month-old, cyclic-light-reared mice by measuring the absorption spectra of regenerated pigments after incubation of retinal homogenates with 11-cis-retinal. All data were normalized to WT levels (Fig. 2A). The absence of GRK1 alone (Grk1 ${ }^{-1-}$ mice) was more deleterious to the retina than the absence of chromophore (Rpe65 $5^{-1-}$ mice), both of these degenerations having been described previously (Redmond et al., 1998; Chen et al., 1999). However, the absence of both GRK1 and RPE65 (Rpe65 $5^{-1-}$ $G r k 1^{-1-}$ mice) exacerbated degeneration, with the opsin levels reduced further compared with $R p e 65^{-1-}$ and $G r k 1^{-1-}$ mice. 
These data were confirmed by Western blot analysis (data not shown). Therefore, opsin phosphorylation, albeit at low levels of only $15 \%$, does provide considerable protection against retinal degeneration.

We analyzed the retinal morphology in 2-month-old WT,

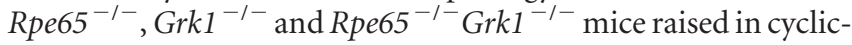
light conditions (Fig. $2 B$ ). The outer segments of rod photoreceptors in WT mice were long and organized. The outer segments were of intermediate length in $R p e 65^{-1-}$ and $G r k 1^{-1-}$ mice, and were short and the least organized in $R p e 65^{-1-} \mathrm{Grk1} 1^{-1-}$ mice. The rows of photoreceptor nuclei in the outer nuclear layer (ONL) were also significantly reduced in Rpe65 $5^{-1-} \mathrm{Grk1} 1^{-1-}$ mice compared with WT, Rpe65 $5^{-l-}$, and $G r k 1^{-1-}$ mice $(p<0.005)$ (Fig. $2 C)$. These data further confirm the severe degeneration of retinae lacking both GRK1 and RPE65.

To examine the age-dependency of the degeneration, we compared opsin levels in 1-, 2-, 4-, and 6-month-old mice (Fig. 2D). The Rpe $65^{-1-}$ mice retained a constant level of opsin over 6 months at $\sim 50-60 \%$ of WT. The $G r k 1^{-1-}$ retinae degenerated steadily over the 6 month period, with only $8.5 \pm 1.5 \%(n=3)$ opsin remaining at 6 months of age. In Rpe $65^{-1-} \mathrm{Grk1}{ }^{-/-}$mice, opsin was barely detectable at 4 months, indicating accelerated retinal degeneration. However, given that only $15 \%$ of opsin in the Rpe $65^{-1-}$ mouse is phosphorylated, the severe aggravation of degeneration in Rpe $65^{-1-} \mathrm{Grk1}^{-1-}$ mice was surprising.

\section{Lack of GRK1 causes retinal degeneration even in the absence of $\alpha$-transducin}

The slow retinal degeneration in RPE65-deficient mice $\left(R p e 65^{-l-}\right.$ ) is caused by the constant activation of transducin by bare opsin, which contains 10-20\% mono-phosphorylation, and degeneration can be suppressed by deleting the $\alpha$-subunit of rod transducin (i.e., the Rpe65 $5^{-1-}$ Gnat $1^{-1-}$ mouse) (Woodruff et al., 2003). To determine whether the rapid degeneration in Rpe $65^{-1-} \mathrm{Grk1}^{-1-}$ mice is due to increased activation of the transducin pathway by fully unphosphorylated opsin, we generated and examined the Rpe65 $5^{-1-}$ Grk1 $1^{-1-}$ Gnat $1^{-1-}$ mouse. The deletion of $\alpha$-transducin alone had no adverse effects on retinal morphology and the opsin levels in 2-month-old Gnat $1^{-1-}$ mice were comparable to WT, as reported previously (Fig. 3A) (see also Calvert et al., 2000). Surprisingly, blocking the transducin pathway only partially rescued the degeneration in $R p e 65^{-1-}$ Grk1 ${ }^{-1-}$ Gnat $1^{-1-}$ mice as the opsin level at 2 months of age improved only to $54.2 \pm 4.9 \%(n=3)$ of WT (vs $\sim 10 \%$ in Rpe $65^{-1-}$ Grk1 $1^{-1-}$ mice). Furthermore, at 4 and 6 months of age, this degeneration was accelerated (Fig. $3 B$ ). Examination of the ONL thickness and outer segment length in 6-month-old Rpe65 $5^{-1-} \mathrm{Grk1}^{-1-} \mathrm{Gnat1}^{-1-}$ retinae compared with age-matched Rpe $65^{-1-} \mathrm{Grk1}^{-1-}$ and WT retinae (Fig. 3C) confirmed the incomplete rescue of retinal degeneration by the deletion of $\alpha$-transducin. This result is in contrast with the complete block of retinal degeneration by the deletion of $\alpha$-transducin in RPE65deficient mice (Woodruff et al., 2003) and indicates that in the absence of GRK1, degeneration occurs by a mechanism unrelated to transducin signaling. Indeed, a comparable, light-independent decrease in opsin levels in 2-month-old $G r k 1^{-1-}$ Gnat $^{-1-}$ mice in cyclic light $(75.7 \pm 10.3 \%)$ and in constant darkness $(71.6 \pm$ $8.0 \%$ ) was observed (Fig. $3 A$ ), supporting this notion.

\section{ERG responses show severe loss of rod function in the absence} of GRK1

To correlate the levels of the light-evoked rod response in these models, we recorded transcorneal scotopic ERGs from 2-month-

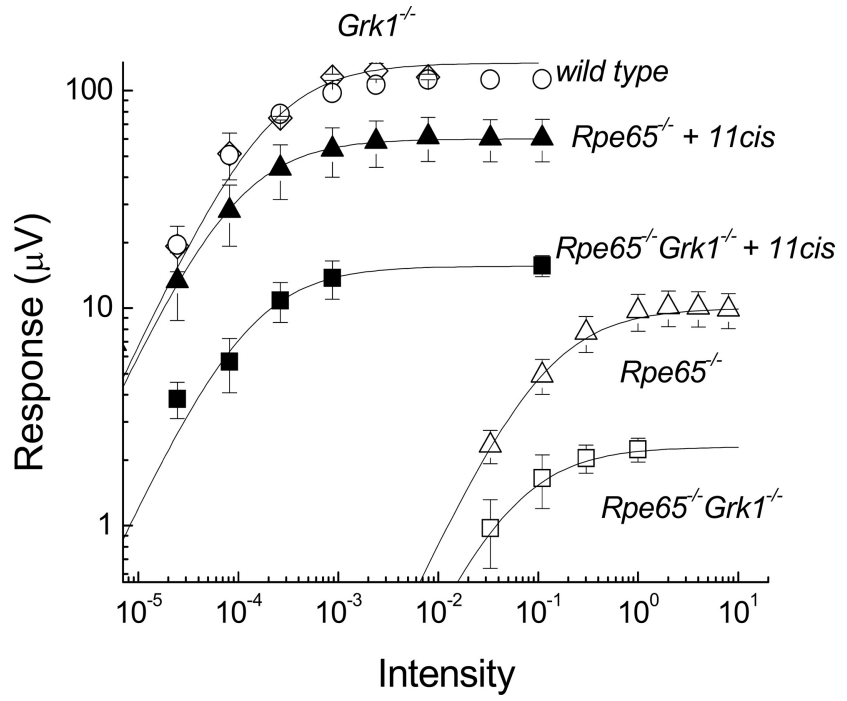

Figure 6. Amplitude of transretinal ERG a-wave as a function of flash intensity in WT (O;

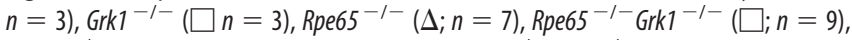
Rpe65 $5^{-1-}$ with 11-cis-retinal $(\mathbf{\Delta} ; n=9)$, and Rpe65 $5^{-1-}$ Grk1 $1^{-1-}$ with 11-cis-retinal ( $n=6)$ retinae. The intensity-response data are fitted by Equation 1. Data from Rpe65 $G r k 1^{-1-}$ mice are from animals raised in cyclic-light conditions. Data are presented as mean \pm $\operatorname{SEM}(n=8)$.

old animals (Fig. 4). Previous studies from Rpe $65^{-1-}$ mice have shown that their cones have almost completely degenerated by that age (Rohrer et al., 2005; Znoiko et al., 2005) and their small ERG responses are triggered by rods (Seeliger et al., 2001). The Rpe65 $5^{-1-}, \mathrm{Grk1}^{-1-}$ Rpe65 $5^{-1-}$ and Rpe65 $5^{-1-} \mathrm{Grk1}^{-1-} \mathrm{Gnat1}^{-1-}$ mice all had undetectable rod a-waves (Fig. 4) and reduced or undetectable b-waves compared with WT mice, in the order of progressive severity. This reduction in sensitivity is consistent with the large accumulation of opsin in the absence of RPE65, which should lower quantum catch and also produce constitutive activation of the transduction cascade by opsin. These results demonstrate that, consistent with our morphological studies above, rod function was more severely affected in $R p e 65^{-1-}$ $G r k 1^{-1-}$ mice compared with Rpe65 ${ }^{-1-}$. However, transcorneal ERG recordings did not allow us to distinguish between the effect of opsin-phosphorylation loss and that of retinal degeneration in reducing rod function.

We therefore initially attempted single-cell, suction-pipette recordings. Unfortunately, the combination of severe degeneration and slow response-termination in $R p e 65^{-1-} \mathrm{Grk1} 1^{-1-}$ rods (Fig. 5, Table 1) made these recordings impossible. Instead, we used ERG recordings from isolated whole retina to assess rod function. We isolated the photoreceptor component (a-wave) of the ERG response by pharmacologically blocking synaptic transmission in the retina, thus eliminating all other components (see Materials and Methods for details). In principle, the a-wave response to a saturating flash contains a dominant rod- and a minute cone-component (Wang et al., 2009). However, the negligible cone component, together with the rapid cone degeneration in the absence of RPE65, allowed us to treat the a-wave as coming purely from rods.

We found no reduction in the saturated response amplitude and in the sensitivity (defined as the reciprocal of the halfsaturating flash intensity) of 1-month-old, dark-reared Grk1 ${ }^{-1-}$ mouse rods compared with those from cyclic-light-reared WT mice (Fig. 5, compare $A, B$; see also Fig. 6, Table 1 ), suggesting limited degeneration at this age (Chen et al., 1999). The response- 
Table 1. Parameters of transretinal ERG recordings

\begin{tabular}{|c|c|c|c|c|}
\hline Genotype & $R_{\max }(\mu \mathrm{V})$ & Sensitivity $\left(I_{o}\right)$ & $T_{\text {peak }}(\mathrm{ms})$ & $\tau_{\text {rec }}(\mathrm{ms})$ \\
\hline Wild type (DL, $n=3$ ) & $112 \pm 2$ & $(1.1 \pm 0.1) \times 10^{-4}$ & $179 \pm 2$ & $414 \pm 39$ \\
\hline $\mathrm{Grk}^{-I-}(\mathrm{DD}, n=3)$ & $118 \pm 9$ & $(1.4 \pm 0.4) \times 10^{-4}$ & $159 \pm 11$ & $17,871 \pm 4910$ \\
\hline Rpe65 $^{-1-}(\mathrm{DL}, n=7)$ & $11 \pm 2$ & $(1.4 \pm 0.3) \times 10^{-1}$ & $111 \pm 5$ & $111 \pm 10$ \\
\hline Rpe65 $5^{-1-} \mathrm{Grk1}^{-1-}(\mathrm{DL}, n=9)$ & $2.2 \pm 0.2$ & $(8.3 \pm 2.5) \times 10^{-2}$ & $231 \pm 41$ & $17,610 \pm 2216$ \\
\hline Rpe65 $^{-1-} \mathrm{Grk1}^{-1-}(\mathrm{DD}, n=8)$ & $2.7 \pm 0.5$ & $(1.8 \pm 0.8) \times 10^{-2}$ & $241 \pm 84$ & $22,051 \pm 8516$ \\
\hline Rpe65 $^{-1-}(\mathrm{DL}, 11$ cis, $n=9)$ & $61 \pm 13$ & $(1.6 \pm 0.4) \times 10^{-4}$ & $190 \pm 12$ & $251 \pm 17$ \\
\hline Rpe65 $^{-1-}$ Grk1 $^{-1-}(\mathrm{DL}, 11$ cis, $n=6)$ & $15 \pm 2$ & $(3.9 \pm 1.1) \times 10^{-4}$ & $192 \pm 19$ & $21,522 \pm 5314$ \\
\hline Rpe65 $5^{-1-}$ Grk1 $^{-1-}(\mathrm{DD}, 11$ cis,$n=5)$ & $12 \pm 1$ & $(3.7 \pm 0.6) \times 10^{-4}$ & $171 \pm 7$ & $27,941 \pm 9207$ \\
\hline
\end{tabular}

Mice were reared in constant darkness (DD) or cyclic light (DL). "11cis" indicates application of 11-cis retinal before recordings. Sensitivity was determined as described in Materials and Methods. $R_{\text {max }}$, Maximal (saturating) response of a-wave; $T_{\text {peak }}$, time to peak; $\tau_{\text {rec }}$, recovery time constant. Values are mean \pm SEM.

recovery time constant in Grk1 ${ }^{-1-}$ mice increased 43-fold compared with WT mice (Table 1), as expected from the critical role of GRK1 in response termination (Chen et al., 1999).

The saturated response amplitude in $R p e 65^{-1-}$ mice showed a 10-fold decrease from WT (Fig. 5, compare $A, C$; see also Fig. 6, Table 1). In addition, their response kinetics were significantly accelerated (Table 1), and their sensitivity declined $>10^{3}$-fold (Fig. 6, Table 1). To determine the role of opsin in this decline of rod function, we incubated $R$ pe $65^{-1-}$ retina with exogenous 11cis-retinal before recordings to convert free opsin into visual pigment. After this treatment, the Rpe $65^{-1-}$ a-wave increased by 5.5 -fold in saturated amplitude and by 880 -fold in sensitivity, and showed significant slowing of its kinetics (Fig. 5, compare $C, E$; see also Fig. 6, Table 1). These changes represent the effect of bare opsin on rod function in Rpe65 $5^{-1-}$ mice (Dizhoor et al., 2008). Notably, both saturated amplitude and sensitivity of the Rpe $65^{-1-}$ a-wave did not recover to WT levels after treatment with 11-cis-retinal (Fig. 6; Table 1), consistent with residual effects due to the shortening of rod outer segments (Fig. 2C) and the reduced opsin levels (Fig. $2 \mathrm{~A}$ ) in the Rpe $65^{-1-}$ retina.

Consistent with their more severe retinal degeneration (Fig. 2), the a-wave of the Rpe $65^{-/-}$Grk1 $1^{-/-}$mice showed a further fivefold decrease in saturated amplitude from that of $R p e 65^{-1-}$ (Fig. 5, compare $C, D$; see also Fig. 6, Table 1). Surprisingly, Rpe $65^{-1-} \mathrm{Grk1}^{-1-}$ rods were 1.7 -fold more sensitive than Rpe $65^{-1-}$ rods despite their more severe degeneration (Table 1) and had more than twofold lower opsin content (Fig. 2A). The response kinetics of Rpe $65^{-1-} \mathrm{Grk1} 1^{-1-}$ rods were comparable to those of $G r k 1^{-1-}$ rods (Table 1), indicating that the lack of phosphorylation of the residual visual pigment still dominated response termination. To determine the role of opsin in the decline of Rpe65 $5^{-1-} \mathrm{Grk1} 1^{-1-}$ rod function, we again used 11-cis-retinal. Following regeneration of the rod visual pigment with 11-cisretinal, the Rpe $65^{-1-}$ Grk1 $1^{-1-}$ a-wave increased by 7 -fold in saturated amplitude and by 220 -fold in sensitivity (Table 1 ). This increase in sensitivity is still four times less than the 880 -fold increase in sensitivity of Rpe $65^{-1-}$ rods. This result indicates that the reduction in total free opsin due to degeneration rather than the absence of opsin phosphorylation in $R p e 65^{-/-} \mathrm{Grk1} 1^{-/-}$mice is the determining factor for rod sensitivity. Once the opsin activation was removed by 11-cis-retinal, the sensitivity of Rpe65 $G r k 1^{-1-}$ rods was 2.4-fold lower than that of Rpe65 $5^{-1-}$ rods (Table 1), also consistent with the lower level of opsin in the former. Likewise, the saturating response amplitude of $R p e 65^{-1-}$ $G r k 1^{-1-}$ rods was four times lower than that of $R p e 65^{-1-}$ rods and approximately eight times smaller than the saturating response in WT rods (Fig. 6; Table 1), consistent with the severe degeneration of the Rpe65 $5^{-1-} \mathrm{Grk1} 1^{-1-}$ retina.

We conclude that, as a surprising consequence of degeneration, the reduced level of opsin (hence a reduced opsin activity that more than overcomes the consequence of the lack of opsin phosphorylation) in $R p e 65^{-1-} \mathrm{Grk1} 1^{-/-}$retina compared with Rpe $65^{-/-}$retina results in a slight increase in sensitivity. Finally, raising Rpe65 $5^{-1-}$ Grk1 $1^{-1-}$ animals in darkness also produced an approximately fivefold increase in sensitivity (Table 1), presumably by allowing 9-cis-retinal visual pigment to gradually accumulate in the outer segments (Fan et al., 2003). Following treatment with 11-cis-retinal, there was no significant difference in the function of rods from Rpe65 $5^{-1-} \mathrm{Grk1} 1^{-1-}$ animals raised in darkness or in cyclic light (Table 1), indicating that light exposure did not affect the extent of degeneration.

\section{Discussion}

The relatively slow rod photoreceptor degeneration in $R p e 65^{-1-}$ mice has been something of a puzzle. A similar degeneration is noted in the $\mathrm{Lrat}^{-1-}$ mouse, also lacking 11-cis-retinal. As both models show a low but constant level of opsin phosphorylation, we designed these experiments to determine whether GRK1 is the kinase involved and whether its removal would accelerate the rod photoreceptor degeneration. Our data show that GRK1 is indeed the kinase underlying opsin phosphorylation in Rpe65 $5^{-1-}$ mice and this phosphorylation apparently provides some protection against degeneration. The surprising result is the large extent of

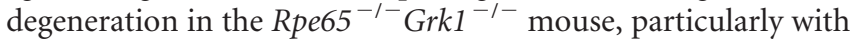
time.

It is well established that GRK1 phosphorylates serines and threonines in the $\mathrm{C}$ terminus of activated rhodopsin and this phosphorylation initiates the rhodopsin inactivation of the visual cascade (Maeda et al., 2003). Our current study shows, however, that GRK1 is capable of phosphorylating rod opsin in the Rpe $65^{-1-}$ mouse even in the absence of light. Presumably, the constitutively active opsin in the absence of chromophore (Fan et al., 2005) is in a form that is weakly recognized and acted upon by the kinase.

Photoreceptor degeneration is greatly accelerated in the Rpe65 ${ }^{-1-}$ $G r k 1^{-1-}$ mouse when compared with the Rpe $65^{-1-}$ mouse, as measured by morphology, opsin levels, and photoreceptor function. Our original hypothesis was that the phosphorylation, even at only a $10-20 \%$ level, would provide some protection against degeneration in the Rpe $65^{-1-}$ mice, presumably by inactivating opsin and thus preventing the continuous activation of transducin. The lack of phosphorylation should increase the overall activity of opsin, but the high level of degeneration, particularly as the animal aged, was unexpected.

Several lines of evidence indicate that the more severe degeneration in the Rpe $65^{-1-}$ Grk1 $1^{-1-}$ mice compared with Rpe65 $5^{-1-}$ mice cannot be explained solely by the increase in opsin activity in the absence of phosphorylation. As only $\sim 15 \%$ of opsin is phosphorylated in the Rpe65 $5^{-1-}$ rods, deletion of GRK1 would result in a corresponding $15 \%$ increase of unphosphorylated op- 
$\sin$. Thus, even if phosphorylated opsin is completely inactive, the opsin activity would be expected to increase at most by a modest $15 \%$ upon deletion of GRK1. In addition, our biochemical studies demonstrated that the opsin content in Rpe65 $5^{-1-}$ Grk1 ${ }^{-1-}$ mice is only one third that of Rpe65 $5^{-1-}$ mice. Thus, the opsin activity in Rpe65 $5^{-1-}$ Grk1 $1^{-1-}$ mice would be expected to be lower based on their substantially reduced opsin content compared with Rpe $65^{-1-}$ mice. We conclude that constitutive opsin activity in the absence of chromophore is not the sole mechanism for photoreceptor degeneration in the Rpe $65^{-1-} \mathrm{Grk1} 1^{-/-}$mice. Instead, we hypothesized that the more severe degeneration in the Rpe $65^{-1-}$ Grk1 $1^{-/-}$mice, particularly in the older animals, is due to the lack of GRK1. Our results indicate that the consequences of this defect increase with the age of the animal, which may explain why $G r k 1^{-1-}$-associated degeneration was not observed in a previous study of dark-reared 2-month-old Grk1 ${ }^{-1-}$ mice (Chen et al., 1999).

To test whether the photoreceptor degeneration in Rpe65 $G r k 1^{-1-}$ mice was due to activation of the visual transduction pathway using $\alpha$-transducin, we investigated the $R p e 65^{-1-}$ Grk1 $1^{-1-}$ Gnat $1^{-1-}$ mouse. Degeneration progressed in the Rpe $65^{-1-} \mathrm{Grk1}^{-1-} \mathrm{Gnat1}^{-1-}$ retina over time even though opsin signaling through rod transducin was silenced. In contrast, the Rpe $65^{-1-}$ retina does not degenerate on a Gnat ${ }^{-1-}$ background (Woodruff et al., 2003). The block of signal transduction in the absence of $\alpha$-transducin allowed us to rule out opsin producing activation of the transduction cascade as the source of degeneration in the Rpe $65^{-1-}$ Grk1 $1^{-1-}$ Gnat $^{-1-}$ retina. Therefore, we believe that the degeneration in the triple knockout is due to the deletion of GRK1. This result was confirmed by the lightindependent degeneration in $G r k 1^{-/-}$retina when GNAT1 was deleted.

If phosphorylation, and therefore the binding of arrestin, is eliminated, the opsin could conceivably interact with other G-proteins, triggering other signaling processes that exacerbate retinal degeneration. Multiple interactions of a single G-proteincoupled receptor (GPCR) with a variety of G-proteins have been identified in other GPCR systems (Bouvier, 1990). The mechanism of GPCR activation of G-proteins is highly conserved. Indeed, certain chimeras of rhodopsin and the $\beta_{2}$-adrenergic receptor have been shown to activate $G_{s}$ (Yamashita et al., 2001; Kim et al., 2005). A second possibility is that GRK1 has a role not yet discerned that could involve the phosphorylation of a protein other than opsin, thus affecting another signaling pathway. Indeed, Carman et al. (1999) have pointed out that GRK may well have other interactions in addition to phosphorylation in G-protein signaling cascades. Deletion of GRK1 may change the expression of other critical proteins. This area certainly merits further investigation. Finally, although Hao et al. (2002) have proposed an apoptotic pathway in light-induced retinal degeneration that does not involve transducin, the pathway observed in our studies is light independent.

Surprisingly, despite their more severe degeneration, Rpe65 $5^{-1-}$ $G r k 1^{-1-}$ rods were more sensitive than $R p e 65^{-1-}$ rods. Our biochemical results demonstrated that the $R p e 65^{-1-}$ rods have threefold higher opsin content than Rpe65 $5^{-1-} \mathrm{Grk1} 1^{-1-}$ rods. This difference in opsin levels will affect sensitivity in two ways. On the one hand, their higher opsin level will result in higher quantum catch, increasing the sensitivity of Rpe $65^{-1-}$ rods compared with Rpe65 $5^{-1-}$ Grk1 ${ }^{-1-}$ rods. On the other hand, however, their higher opsin level will also result in higher opsin activity, decreasing the sensitivity of $R p e 65^{-1-}$ rods compared with Rpe $65^{-1-}$ Grk $1^{-1-}$ rods. The lower sensitivity of $R p e 65^{-1-}$ rods indicates that the desensitization due to opsin activity outweighs the increase in quantum catch. This result is consistent with the nonlinear relation between loss of quantum catch and reduction in sensitivity following a bleach (Jones et al., 1996; Luo and Yau, 2005). Thus, as a surprising consequence of the more severe degeneration in Rpe $65^{-1-}$ Grk1 $1^{-1-}$ mice compared with Rpe $65^{-1-}$ mice, the total opsin activity was actually lower in the double knock-out. Treatment with exogenous chromophore will result in increased sensitivity first, by increasing quantum catch and second, by removing opsin activation. We found that following treatment with exogenous chromophore the sensitivity of the Rpe $65^{-1-} \mathrm{Grk1} 1^{-1-}$ rods was 2.5 -fold lower than that of the Rpe $65^{-1-} \mathrm{Grk1}^{-1-}$ rods, consistent with the relative levels of opsin in the two lines. The similarity between decline in opsin content and reduction in sensitivity is consistent with only a minor role for the activity of the additional 15\% unphosphorylated opsin in the double knockout.

In summary, several conclusions can be drawn from this study. First, the phosphorylation of opsin in $R p e 65^{-1-}$ mice is due to the action of GRK1. Second, this phosphorylation is protective against the retinal degeneration caused by opsin activity. Third, deletion of GRK1 contributes to the increasing retinal degeneration with age in Rpe65 $5^{-1-} \mathrm{Grk} 1^{-1-}$ mice. Finally, the retinal degeneration driven by the lack of GRK1 is through a non-transducin-based signaling pathway.

\section{References}

Ablonczy Z, Knapp DR, Darrow R, Organisciak DT, Crouch RK (2000) Mass spectrometric analysis of rhodopsin from light damaged rats. Mol Vis 6:109-115.

Ablonczy Z, Crouch RK, Goletz PW, Redmond TM, Knapp DR, Ma JX, Rohrer B (2002) 11-cis-retinal reduces constitutive opsin phosphorylation and improves quantum catch in retinoid-deficient mouse rod photoreceptors. J Biol Chem 277:40491-40498.

Adams RA, Liu X, Williams DS, Newton AC (2003) Differential spatial and temporal phosphorylation of the visual receptor, rhodopsin, at two primary phosphorylation sites in mice exposed to light. Biochem J 374:537-543.

Arshavsky VY (2002) Rhodopsin phosphorylation: from terminating single photon responses to photoreceptor dark adaptation. Trends Neurosci 25:124-126.

Batten ML, Imanishi Y, Maeda T, Tu DC, Moise AR, Bronson D, Possin D, Van Gelder RN, Baehr W, Palczewski K (2004) Lecithin-retinol acyltransferase is essential for accumulation of all-trans-retinyl esters in the eye and in the liver. J Biol Chem 279:10422-10432.

Bayer AU, Cook P, Brodie SE, Maag KP, Mittag T (2001) Evaluation of different recording parameters to establish a standard for flash electroretinography in rodents. Vis Res 41:2173-2185.

Bolnick DA, Walter AE, Sillman AJ (1979) Barium suppresses slow PIII in perfused bullfrog retina. Vision Res 19:1117-1119.

Bouvier M (1990) Cross-talk between second messengers. Ann N Y Acad Sci 594:120-129.

Calvert PD, Krasnoperova NV, Lyubarsky AL, Isayama T, Nicoló M, Kosaras B, Wong G, Gannon KS, Margolskee RF, Sidman RL, Pugh EN Jr, Makino CL, Lem J (2000) Phototransduction in transgenic mice after targeted deletion of the rod transducin alpha-subunit. Proc Natl Acad Sci U S A 97:13913-13918.

Carman CV, Parent JL, Day PW, Pronin AN, Sternweis PM, Wedegaertner PB, Gilman AG, Benovic JL, Kozasa T (1999) Selective regulation of Galpha(q/11) by an RGS domain in the G protein-coupled receptor kinase, GRK2. J Biol Chem 274:34483-34492.

Chen CK, Burns ME, Spencer M, Niemi GA, Chen J, Hurley JB, Baylor DA, Simon MI (1999) Abnormal photoresponses and light-induced apoptosis in rods lacking rhodopsin kinase. Proc Natl Acad Sci USA 96:3718-3722.

Chen J, Makino CL, Peachey NS, Baylor DA, Simon MI (1995) Mechanisms of rhodopsin inactivation in vivo as revealed by a COOH-terminal truncation mutant. Science 267:374-377. 
Dartnall HJ (1968) The photosensitivities of visual pigments in the presence of hydroxylamine. Vision Res 8:339-358.

Dizhoor AM, Woodruff ML, Olshevskaya EV, Cilluffo MC, Cornwall MC, Sieving PA, Fain GL (2008) Night blindness and the mechanism of constitutive signaling of mutant G90D rhodopsin. J Neurosci 28:1166211672.

Fan J, Rohrer B, Moiseyev G, Ma JX, Crouch RK (2003) Isorhodopsin rather than rhodopsin mediates rod function in RPE65 knock-out mice. Proc Natl Acad Sci U S A 100:13662-13667.

Fan J, Woodruff ML, Cilluffo MC, Crouch RK, Fain GL (2005) Opsin activation of transduction in the rods of dark-reared RPE65 knockout mice. J Physiol 568:83-95.

Fan J, Rohrer B, Frederick JM, Baehr W, Crouch RK (2008) Rpe65-/- and Lrat - / - mice: comparable models of leber congenital amaurosis. Invest Ophthalmol Vis Sci 49:2384-2389.

Gresh J, Goletz PW, Crouch RK, Rohrer B (2003) Structure-function analysis of rods and cones in juvenile, adult and aged C57BL/6 and Balb/C mice. Vis Neurosci 20:211-220.

Hao W, Wenzel A, Obin MS, Chen CK, Brill E, Krasnoperova NV, EversoleCire P, Kleyner Y, Taylor A, Simon MI, Grimm C, Remé CE, Lem J (2002) Evidence for two apoptotic pathways in light-induced retinal degeneration. Nat Genet 32:254-260.

Hurley JB, Spencer M, Niemi GA (1998) Rhodopsin phosphorylation and its role in photoreceptor function. Vision Res 38:1341-1352.

Jones GJ, Cornwall MC, Fain GL (1996) Equivalence of background and bleaching desensitization in isolated rod photoreceptors of the larval tiger salamander. J Gen Physiol 108:333-340.

Kelleher DJ, Johnson GL (1986) Phosphorylation of rhodopsin by protein kinase C in vitro. J Biol Chem 261:4749-4757.

Kim JM, Hwa J, Garriga P, Reeves PJ, RajBhandary UL, Khorana HG (2005) Light-driven activation of beta 2 -adrenergic receptor signaling by a chimeric rhodopsin containing the beta 2-adrenergic receptor cytoplasmic loops. Biochemistry 44:2284-2292.

Luo DG, Yau KW (2005) Rod sensitivity of neonatal mouse and rat. J Gen Physiol 126:263-269.

Maeda T, Imanishi Y, Palczewski K (2003) Rhodopsin phosphorylation: 30 years later. Prog Retin Eye Res 22:417-434.

Mendez A, Burns ME, Roca A, Lem J, Wu LW, Simon MI, Baylor DA, Chen J (2000) Rapid and reproducible deactivation of rhodopsin requires multiple phosphorylation sites. Neuron 28:153-164.

Newton AC, Williams DS (1993) Rhodopsin is the major in situ substrate of protein kinase $\mathrm{C}$ in rod outer segments of photoreceptors. J Biol Chem 268:18181-18186.
Nymark S, Heikkinen H, Haldin C, Donner K, Koskelainen A (2005) Light responses and light adaptation in rat retinal rods at different temperatures. J Physiol 567:923-938.

Ohguro H, Rudnicka-Nawrot M, Buczylko J, Zhao X, Taylor JA, Walsh KA, Palczewski K (1996) Structural and enzymatic aspects of rhodopsin phosphorylation. J Biol Chem 271:5215-5224.

Redmond TM, Hamel CP (2000) Genetic analysis of RPE65: from human disease to mouse model. Methods Enzymol 316:705-724.

Redmond TM, Yu S, Lee E, Bok D, Hamasaki D, Chen N, Goletz P, Ma JX, Crouch RK, Pfeifer K (1998) Rpe65 is necessary for production of 11cis-vitamin A in the retinal visual cycle. Nat Genet 20:344-351.

Rohrer B, Lohr HR, Humphries P, Redmond TM, Seeliger MW, Crouch RK (2005) Cone opsin mislocalization in Rpe65 $5^{-/-}$mice: a defect that can be corrected by 11-cis retinal. Invest Ophthalmol Vis Sci 46:3876-3882.

Seeliger MW, Grimm C, Ståhlberg F, Friedburg C, Jaissle G, Zrenner E, Guo H, Remé CE, Humphries P, Hofmann F, Biel M, Fariss RN, Redmond TM, Wenzel A (2001) New views on RPE65 deficiency: the rod system is the source of vision in a mouse model of Leber congenital amaurosis. Nat Genet 29:70-74.

Sillman AJ, Ito H, Tomita T (1969) Studies on the mass receptor potential of the isolated frog retina. I. General properties of the response. Vision Res 9:1435-1442.

Wald G, Brown PK (1958) Human rhodopsin. Science 127:222-226.

Wang JS, Estevez ME, Cornwall MC, Kefalov VJ (2009) Intra-retinal visual cycle required for rapid and complete cone dark adaptation. Nat Neurosci 12:295-302.

Williams DS, Liu X, Schlamp CL, Ondek B, Jaken S, Newton AC (1997) Characterization of protein kinase $\mathrm{C}$ in photoreceptor outer segments. J Neurochem 69:1693-1702.

Woodruff ML, Wang Z, Chung HY, Redmond TM, Fain GL, Lem J (2003) Spontaneous activity of opsin apoprotein is a cause of Leber congenital amaurosis. Nat Genet 35:158-164.

Xiong W, Nakatani K, Ye B, Yau K (1997) Protein kinase C activity and light sensitivity of single amphibian rods. J Gen Physiol 110:441-452.

Yamashita T, Terakita A, Shichida Y (2001) The second cytoplasmic loop of metabotropic glutamate receptor functions at the third loop position of rhodopsin. J Biochem 130:149-155.

Znoiko SL, Rohrer B, Lu K, Lohr HR, Crouch RK, Ma JX (2005) Downregulation of cone-specific gene expression and degeneration of cone photoreceptors in the Rpe $65^{-1-}$ mouse at early ages. Invest Ophthalmol Vis Sci 46:1473-1479. 